\title{
LA CENTINELA: UN ASENTAMIENTO INKA-CHINCHA. RASGOS ARQUITECTÓNICOS ESTATALES Y LOCALES
}

Por:

Julián Idilio Santillana

Universidad Nacional Mayor de San Marcos

Este trabajo forma parte de un estudio más amplio e interdisciplinario desarrollado por el Instituto de Investigaciones Andinas de Nueva York en el valle de Chincha, auspiciado por la fundación Tinker. 

La organización Inka es la última manifestación estatal en el desarrollo cultural andino autónomo antes de su introducción en el escenario de la historia universal después de la invasión europea.

A partir de Pachacutec, la expresión expansiva de los Inkas condujo a la incursión y captación de inmensos recursos energéticos con fines de acumulación excedentaria rompiendo al parecer viejos patrones redistributivos de vida comunal imperantes en la América Andina Central.

Una de las regiones componentes del Tawantinsuyo era el Chinchaysuyo que cubría una de las más grandes concentraciones de recursos y un considerable asentamiento de etnias, muchas de ellas expresadas en señoríos y estados. En este territorio se encontraba el reyno de Chincha.

La incursión inicial a este territorio fue por las tropas de Capac Yupanqui sin embargo, la consolidación de la presencia Inka en el valle se logró recién con Tupac Yupanqui, quien implantó en la región "las leyes y organización Inka" (Rostworowski, 1977: 106). De esta manera el reyno Chincha fue asimilado a la estructura incaica ya en las últimas décadas del Tawantinsuyo, como una formación social significativa por el rol que desempeñaba en la organización económica del Imperio. Se manifiesta como un poder local poco antes de 1250 y termina como tal en 1476, cuando los Inkas conquistaron la Costa Sur (Rowe, 1966: 194).

Este reyno era una de las sociedades costeñas más complejas y un centro próspero de mercaderes que traficaban un conjunto de bienes a larga distancia. Cuando los Inkas conquistaron el valle no destruyeron la producción de bienes ni la modalidad de distribución panandina, por el contrario monopolizaron el comercio "actuando el estado Inka como un mercado gigante" (Morris et al. Ms). El caso de Chincha fue único dentro del estado Inka. Aparentemente fue el único núcleo al que se le permitió continuar con esta tradición de comercio, ya que el estado Inka habría anulado deliberadamente muchas de estas actividades en otras regiones donde existían y en otros los asimiló al control directo de su producción y distribución.
Según la documentación escrita, en la época Inka, Chincha comerciaba como parte articulada a otros dos ecosistemas: la región del Collao y la región costeña septentrional del Inkario, logrando así un comercio a larga distancia marítimo y por tierra.

La acumulación excedentaria de bienes se logró tanto por la producción de sectores totalmente especializados concentrados en el mismo asentamiento Chincha como por la captación de otros bienes de otras áreas. El documento colonial de data temprana llamado genéricamente "Aviso" por M. Rostworowski, explicita sobre la naturaleza de los bienes que se comercializaban y el componente poblacional de trabajadores que se encontraban en Chincha: "Existía 6 mil mercaderes que realizaban viajes desde Chincha al Cuzco, por todo el Collao y al norte hasta Quito y Puerto Viejo, de donde traían chaquira de oro y esmeraldas para los curacas del Inca" (Rostworowski, 1977: 108). El comercio básico con el Norte consistía en conseguir Mullu (Spondylus pictorum), un bien de carácter sagrado y codiciado en todo el área andina. Tenía gran demanda y se podía conseguir sólo en las tibias aguas septentrionales. Por otro lado, los mercaderes chinchanos conseguían el cobre del Collao y llevaban al Norte para comerciar por las conchas.

Junto a los mercaderes existían en Chincha pescadores y según los documento en número de 10 mil. Con igual significado económico, existían también en el valle un gran número de agricultores y artesanos.

Pues bien, esta sociedad tan compleja económica y políticamente construyó un sinnúmero de centros poblados, centros ceremoniales y centros de poder a lo largo y ancho del valle de Chincha. Casi todos ellos de orden monumental por sus grandes dimensiones en planta y elevación. El más grande y complejo de estos asentamientos es La Centinela y según Menzel y Rowe (1966) se trataría del complejo arquitectónico Chincha más grande e importante y asiento "del señor de Chincha", formando una unidad con La Cumbe y Tambo de Mora. Al costado de este complejo se levantó el centro administrativo estatal Inka mas importante en el valle. 
De esta manera encontramos evidenciados arqueológicamente la ocupación local chinchana y la estatal inka, cada una de ellas por separado. Sin embargo, hay áreas de ocupación en La Centinela que presentarían evidencias culturales de ambas formaciones.

Nuestra preocupación en esta oportunidad se centra en analizar algunos rasgos del patrón arquitectónico de La Centinela, determinar algunos de los elementos formales constitutivos de la capital chinchana; determinar el área real de ocupación inka; ver cuantos y cuales, entre los demás sectores son construidos y ocupados por los Chincha, los Inkas o por ambos.

La Centinela.- En el valle comprendido entre el litoral marino y el inicio de las primeras estribaciones andinas de la cordillera occidental por un lado y flanqueado por los bancos de arena ligeramente elevados por el Norte y Sur se extiende una extensa área irrigada que se denomina el valle de Chincha al Norte del departamento de Ica, al Sur del Perú. Es un típico valle costeño que irrumpe el arenal desértico característico del gran sistema andino intertropical (Dollfus 1980).

Toda el área estaba ocupada durante el Intermedio Tardío y el Horizonte Tardío. Aparentemente, no había necesidad de ampliar la base ecológica regional, ya que los testimonios arqueológicos (Menzel y Rowe, 1966 y Menzel, 1966) indican -en general- la ocupación del reyno Chincha solo en el valle e incluso cuando los Inkas se asentaron en la zona, pudo sostener las necesidades del Estado.

Después de nuestro reconocimiento del valle y el estudio del plano publicado por Wallace (1971), los sitios que se encuentran al lado Oeste del valle estarían agrupados en torno a dos complejos, ambos próximos al mar y asociados a dos ríos, el complejo Norte vinculado al río Chico y el complejo Sur al río Matagente. El primer complejo tendría como ejes centrales a La Centinela, La Cumbe y Tambo de Mora: mientras que el segundo a $10 \mathrm{~km}$ al Sur tendría su eje central en La Centinela de San Pedro. En torno a estos dos complejos se aglutinan un conjunto de montículos de diversos tamaños y diversos componentes culturales. Si bien estas matrices se observan con claridad para la ocupación tardía, es probable que este patrón tenga una tradición anterior que hoy todavía no conocemos.

El complejo de La Centinela tiene en la actualidad una extensión de 600 x 500 m, cubriendo un área aproximada de $30 \mathrm{Ha}$. Las pirámides suman 11, todas ellas claramente definidas y separadas resaltando la huaca central cuya altura sobrepasa los $43 \mathrm{~m}$, y de acuerdo a nuestra sectorización corresponde al sector II.

En el caso de La Centinela resulta ventajosa la manifestación de la arquitectura sobre la superficie ya que nos permite visualizar la mayoría de sus elementos y es relativamente fácil definir los componentes arquitectónicos por los rasgos homogéneos que le dan organicidad a los espacios. Las calles, muros divisorios y las plazas son los elementos que van separando articuladamente las unidades arquitectónicas.

Siguiendo los indicadores señalados, encontramos conglomerados arquitectónicos de distintos tamaños y diversa complejidad. Los conglomerados mayores se denominan sectores, estos a su vez se dividen en subsectores y solo en un caso los subsectores en unidades de subsectores (Ver plano 1).

\section{La Estructura Interna y el Nucleamiento Múl-} tiple.- El conjunto de pirámides o sectores de La Centinela obedecen a una configuración de carácter nucleado y no disperso. Sin embargo, este carácter nucleado no representa una horizontalidad cultural espacial ni cronológica, por el contrario es multicomponente al evidenciarse por lo menos dos ocupaciones.

Pero estos niveles de ocupación cultural distintos no han significado falta de una imbricación arquitectónico-urbana, por el contrario, existe un nivel orgánico articulado que refleja una planificación en la concepción de los conglomerados y una dirección organizada en las construcciones.

La planificación como criterio organizado y sistemático en el valle chinchano no es un 
criterio que se da recién con la conquista Inka, sino mas bien es una modalidad técnica que existía con anterioridad y refleja el nivel de complejidad de la formación social chinchana. Lo que sucede es cuando los Inkas llegan al valle, organizan "su espacio" en La Centinela con criterios de planificación estatales y -al parecer- obliteran ciertos conceptos formales en algunos sectores de ocupación chinchana, de acuerdo a los conceptos y necesidades también estatales, pero en ningún caso rompen el carácter nucleado y múltiple de pirámides cerradas que domina el conjunto.

El Sector Inka III.- Se levanta en el extremo Sur-Oeste del complejo y ocupa un área mayor a la que ocupan cada uno de los otros sectores componentes de La Centinela. Su estructura interna se inserta dentro de dos cuerpos o bloques. El primero es un bloque grande cuyo espacio alberga a un conjunto de construcciones que corresponden al edificio principal y presumiblemente relacionado en forma más directa al Inka. El segundo es un bloque cuyo espacio define una gran plaza y dos pequeños recintos. La plaza es la más grande en todo el conjunto. Si bien los dos cuerpos forman una unidad espacial arquitectónica, estos están separados por una callejuela que cruza el complejo de Sur a Norte. El sector Inka se levanta en tres niveles o plataformas, sin embargo la elevación total no alcanza la altura del más pequeño de los sectores de ocupación chinchanas.

El conjunto de edificios o recintos se encuentran aglutinados formando pequeños conglomerados, llamados subsectores por nosotros. Con excepción del IIIC los demás subsectores están organizados en torno a tres elementos: recintos, plazas y accesos. Estos son los indicadores de la trama característica de los Inkas. La plaza es el elemento indicador y aglutinante de la arquitectura Inka, y parecería definir en La Centinela el carácter público o privado del espacio en la medida en que las plazas grandes habrían servido para congregar a grandes sectores poblacionales, no así las pequeñas a manera de plazoletas, que tienen accesos únicos y restringidos y, que en este caso están asociados a recintos de factura fina que indudablemente representan unidades muy especiales.
En el caso Inka tenemos tres plazas que tienen accesos directos del exterior (IIIA, IIID y IIIF) y aparentemente asociados y algunos recintos grandes; dos de tamaño mediano (IIIA y IIIE) que muestran ya una cierta restricción y exclusividad en el acceso y los pequeños (IIIA y IIIB) asociados a recintos de fino paramento con un solo acceso a través de dos vanos de doble jamba y escalinatas. Sin embargo, este modelo de aglutinamiento constructivo en torno a una plaza no se aplica en el subsector IIIC donde el eje de articulación es un corredor.

De acuerdo a las evidencias que hoy se exhiben y que no han variado sustantivamente de su trazo original, el subsector IIID presenta el ejemplo mas claro del clásico patrón Inka, en el que en torno a una plaza se levantan un conjunto de construcciones. Es la clásica cancha Inka. Las cuatro construcciones que circundan el lado Norte son cuadradas, están encerradas a lo largo por un muro que define un pasaje y forma a su vez la única entrada existente para estos recintos. Por otro lado, las construcciones del lado Oeste y sobre todo del Este son mas grandes y simétricas a manera de grandes galpones o kallankas de los asentamientos Inkas de administración provincial (Morris 1974) y habrían servido para el alojamientos de distintos sectores poblacionales que llegaban a este centro administrativo. Merece destacar también que los muros adosados formando muros dobles han servido en muchos casos para definir los límites de cada uno de estos subsectores. ¿Refleja esto una planificación por separado? Probablemente sí, ya que la construcción refleja volúmenes agregados unos a otros. Esta manera de planificación y construcción se evidencia también en cuanto a la separación Inka de la Chincha, como es el caso del límite Norte del subsector Inka IIIA y el sector II de ocupación local; de la misma manera en la división del subsector IIIF Inka y el sector $\mathrm{V}$ chinchano.

Reafirmando la trama Inka de las distintas construcciones de la arquitectura de poder, religiosa y doméstica en otros complejos los subsectores IIIA y IIIB residenciales reproducen el patrón constructivo que gira en torno a un espacio abierto, en este caso pequeños donde la orientación de los recintos principales y de los vanos están al Oeste, con una magnífica mira- 
da al mar. Los recintos son pequeños y exclusivos cuyos atributos arquitectónicos son distinguibles en cada uno de los edificios. Si bien la plaza aglutina, los recintos están construidos también como espacios cerrados de funciones específicas. Las construcciones guardan regularidad en sus disposiciones, existe una unidad volumétrica y de conjunto sin romper el equilibrio constructivo entre el tamaño y la forma.

En el caso del IIIA, el conjunto de los recintos tienen dos cuerpos y distintas orientaciones que indicarían variación en la importancia y consecuentemente en la función de los mismos. El primer núcleo, dispuesto en torno a la plaza, orientado al Oeste correspondería al área más importante. El segundo núcleo de edificios ubicados detrás del primero, dispuesto frente a la plaza mediana, correspondería a un segundo en orden de importancia, que en todo caso parece tratarse de un área complementaria de ciertos servicios para los ocupantes del primer núcleo.

En cuanto al subsector IIIB la trama formal es más simple y mucho más evidente el carácter exclusivo y rígido. Está compuesto por tres recintos cuyas puertas dan a un corredor que viene a ser la única entrada. Todo esto hace que los recintos se presenten en una cierta modalidad de cautivos en la trama arquitectónica.

Los Sectores de Ocupación Local.- No todos los sectores de ocupación chinchana exhiben construcciones en el porcentaje suficiente que haga posible ver la trama, ni todos ellos están bien conservados, razones por las cuales tomamos para la descripción aquellas unidades cuyas evidencias arquitectónicas sean visibles y el estado de conservación relativamente bueno. Las dimensiones de estos sectores varían, habiendo unidades grandes (I y II) medianas $(\mathrm{V}, \mathrm{VI}$ y $\mathrm{X}$ ) y pequeñas, mas bien por lo que de ellos quedan (IV, VII y X).

Los sectores I y II son los que se encuentran orientados al Oeste y adosados al área de ocupación Inka. Estos dos sectores como los demás, están definidos como montículos a manera de pirámides. La altura de cada una de estas pirámides varía reflejando una correspondencia entre la extensión perimétrica y la elevación. Esto sugiere también la relación existente entre la dimensión y la jerarquía de los sectores. El más grande en su extensión y elevación es el montículo mayor y central denominado sector II, en el que además predominan los atributos de monumentalidad y algunos rasgos arquitectónicos típicos de una estructura de poder.

En ningún caso hay edificios en el primer nivel, escasamente en los niveles intermedios y en mayor número en la plataforma final mas elevada. Aquí los recintos se encuentran aglutinados.

A diferencia del sector Inka los sectores locales tienen un patrón diferente en la concepción del espacio, de los volúmenes y la articulación de los distintos elementos arquitectónicos. Por ejemplo, la plaza o espacio abierto es un elemento ausente entre los Chincha. Si bien en los sectores de ocupación local de La Centinela hemos identificado solamente dos plazas (I y II) creemos que se trata de uno de los elementos de innovación que los Inkas introdujeron en el valle. La presencia Inka en este complejo obliteró antiguos conceptos y patrones para darle un nuevo carácter a la concepción del espacio, al desarrollo plano y horizontal de las construcciones, en oposición a las pirámides chinchanas.

La introducción de nuevos elementos o estructuras implican también una nueva trama que rompe los conceptos de planificación existentes entre los Chincha y logran una imbricación compatibilizando dos patrones y dos conceptos. De allí, que en los casos de los sectores Chincha señalados surgen construcciones de distintos tamaños en torno a las plazas.

Destacan también en las pirámides chinchanas, la presencia de pequeños recintos construidos en la última plataforma superior y que están generalmente asociados a dos pequeños espacios abiertos que recuerdan los "patios traseros" (ver plano). Este pequeño número de recintos aglutinados, más algunos otros recintos que existen en algunas plataformas intermedias, indicarían que el número de ocupantes era reducido en relación a la gran extensión de área construida. Obviamente debe haber correspondido a sectores poblacionales 
pequeños y privilegiados. De hecho, algunas de estas construcciones tenían funciones domésticas por las evidencias de carbón, semillas y en general material orgánico que aparecen en los basurales ubicados en lugares marginales al área nuclear de las construcciones. A diferencia de los otros sectores Chincha, la trama constructiva de la pirámide central, sector II, es distinta y más compleja. Si bien la forma es de un rectángulo regular, de muros de cerramientos altos y anchos y de plataformas superpuestas que desarrollan en niveles convergentes, varían en cuento a espacios constructivos y a la planificación del conjunto en sí.

$\mathrm{Al}$ parecer, en el subsector IID se concentran los recintos más significativos de ésta pirámide, lamentablemente hoy solo quedan pedazos de frisos en alto relieve de diseño típicamente chinchano.

Finalmente, corona esta pirámide mayor una pequeña plataforma que viene a ser la cima del sector. En la actualidad hay una cruz católica que mira al mar. Esta plataforma correspondería a una parte del conjunto ceremonial mayor.

Los Sectores de Ocupación Bilateral.- Los sectores VIII y XI, merecen una especial referencia puesto que poseen un conjunto de indicadores que sugieren conceptos especiales en la planificación y construcción. Tienen un conjunto de elementos tanto chinchanos como Inka que hacen pensar en unidades que contienen formas estatales y locales. Puede tratarse de una ocupación estrictamente local que adopta conceptos y elementos Inka y locales o, puede también tratarse de sectores de ocupación dual.

Tanto en el sector VIII como en el XI se encuentran espacios abiertos o plazas como componentes básicos en la estructura de la unidad, aunque como plaza aparece mejor definida en el sector VIII. En el sector XI el "espacio abierto" es de forma irregular, emplazado de Oeste a Este sobre el cual se alinean las construcciones.

Otra de las características Inka estatales que se encuentran en estos sectores es la presencia de grupos de grandes recintos rectangulares construidos en torno a las plazas. Estos elementos Inka contrastan fuertemente con los pequeños recintos cuadrados o rectangulares que dominan la concepción arquitectónica local chinchana.

Por otro lado también es evidente la presencia de conceptos totalmente locales en la planificación y construcción de estos sectores. En ambos casos se trata todavía de sectores de un patrón piramidal en base a la superposición de pequeñas y angostas plataformas para lograr en la cima una pequeña terraza donde se nuclearizan las edificaciones. Estas elevaciones a manera de montículos tampoco se manifiestan en el área total del sector, más bien se reduce a una pequeña área o subsector donde se reproduce la trama local de pequeños recintos y "los patios traseros".

Los elementos típicos de vanos, accesos y materiales constructivos. Consecuentemente, en estos dos sectores se evidencia un gradual replanteamiento de formas combinadas sobre una tradición arquitectónico-urbana dominante, en la perspectiva de un nuevo acomodamiento formal de acuerdo a las nuevas necesidades de la administración estatal Inka.

Componentes Estatales y Locales.- El examen de la arquitectura y trama urbana de los sectores de ocupación chinchana, del sector Inka y los otros dos sugeridos como de ocupación bilateral, reflejan simultáneamente algunos elementos comunes por tratarse de edificaciones construidas dentro de los marcos diseñados por estructuras de organización complejas. Estos elementos serían: 1.- Que cada uno de los sectores fueron construidos dentro de los estilos de arquitectura residencial y administrativa. 2.- Como consecuencia del primero, estos sectores fueron construidos para pequeños sectores poblacionales directamente participantes de la estructura de poder y 3.- Que la construcción de estos sectores se hicieron con el concurso de una fuerza de trabajo supracomunal.

En consecuencia se produce la estandarización como mecanismo que generaliza un conjunto de elementos arquitectónicos y de for- 
mas urbanísticas hasta lograr que los sectores se vean concordantes con una planificación formal preconcebida. Pues entonces, en uno y otro caso, se tratan casi de los mismos elementos que componen la manifestación estatal Inka y local chinchana, encontrando mayores diferencias en las formas, frecuencias y tamaños y poco en el concepto. Sin embargo, cuando se trata de la trama urbanística los conceptos de los que parten son sustantivamente diferentes, como hemos descrito líneas arriba.

En cuanto al concepto subyacente en los rasgos arquitectónicos y en ciertos casos en los rasgos de la trama urbano-estatales el patrón característico e inconfundible es la forma trapezoidal. El concepto trapezoidal en el sector Inka de La Centinela se manifiesta en las siguientes formas: a.- En la forma del emplazamiento de los cuerpos, uno grande y otro chico que compone la traza Inka. El primer grupo compuesto por los subsectores IIIA, B, C, D y $\mathrm{E}$; y en el segundo cuerpo definido por el subsector F. b.- en la forma de los siguientes elementos arquitectónicos: puertas, hornacinas y probablemente ventanas. c.- En la forma de los muros y en el perfil volumétrico de los subsectores. En general, todas las construcciones estatales a lo largo del Tawantinsuyu y, en este caso, los muros del sector Inka de La Centinela tienen la forma trapezoidal.

En el caso de la ocupación chinchana los componentes son totalmente distintos. En contraste con el diseño trapezoidal de los Inkas, la arquitectura y la trama urbana se manifiestan en formas cuadrangulares o rectangulares. Aún cuando sugeríamos líneas arriba que la plaza o espacio abierto, en la composición urbana local, sea un elemento adquirido o impuesto por los Inkas, estos no reproducen la forma trapezoidal, por el contrario en los casos en que logramos identificarlas perviven las formas cuadradas o rectangulares.

En contraste con las puertas Inka, los locales son de una sola variedad consistente en una forma simple rectangular, mientras que los Inkas representan dos variedades, definidas por la composición de sus elementos y no porque sean productos de conceptos distintos. Ambos son de forma trapezoidal que se diferencian entre sí porque una tiene doble jamba y la otra no. La presencia y frecuencia de ésta está en rela- ción a la importancia del área. En el caso de La Centinela se encuentran en las entradas a los subsectores importantes y en ningún caso están emplazadas como puertas de recintos, a estos se ingresan a través de puertas de una sola jamba.

La casi totalidad de puertas de doble jamba se encuentran en los subsectores IIIA y IIIB de componentes habitacionales. La única puerta con estos atributos fuera de estos núcleos se encuentran en el acceso Este del sector IIIC, al parecer una zona de componentes económicamente significativos.

En cuento se refiere a las hornacinas, las de factura local presentan también grandes diferencias con los Inkas. En comparación con las estatales, que son trapezoidales, de buen acabado y textura regular, los locales son más pequeños, ligeramente cuadrados o rectangulares y de acabados no siempre finos. Las hornacinas locales se encuentran en algunos muros de los recintos pequeños y en pequeños espacios abiertos. La construcción en cada una de las culturas obedecen a dos criterios distintos: Mientras en el caso Inka las hornacinas se levantan a medida que se van construyendo los muros como elementos integrantes del paño, en el caso local son una especie de "agregados" que se hacen después de terminado el muro. Aparentemente los Chincha las "construyen" desgastando una pequeña área en el paño hasta darle una forma cuadrada o rectangular.

La presencia de estas dos formas de hornacinas se encuentra, en principio, por separado tanto en el sector Inka como en los sectores chinchanos. Sin embargo, en la plaza mediana del subsector IIIA, de ocupación Inka encontramos ambas formas: la local y la estatal. ¿Por qué se presentan estas dos formas en una plaza tan importante del sector íntegramente estatal? ¿Es muestra de la presentación del trabajo rotativo que los Chinchanos debían al estado Inka? iSe trataría de un espacio que en determinados acontecimientos era mutuamente compartido por los Inkas y Chinchas? Era muestra del rango que prodigaba el estado a los "Señores de Chincha".

Consolidando nuestro planteamiento sobre la ocupación bilateral de algunos sectores de La Centinela, encontramos que en el sector XI, las formas trapezoidales de las hornacinas 
son frecuentes aunque hay algunas que aparentan un diseño local. Las únicas hornacinas del sector se encuentran en el muro interior Norte del recinto grande del subsector XIA. En total son 17; 9 en el lado derecho y 8 en el izquierdo. La parte central del muro, en una extensión de $3 \mathrm{~m}$ no presenta hornacinas. Los tamaños son desiguales y tienen una distribución asimétrica. Si bien la forma trapezoidal es la dominante hay algunas que difieren, insinuando más bien una forma cuadrada. No están construidas en línea recta ni a la misma altura. La segunda hornacina, empezando del extremo derecho del paño, evidencia haber sido originalmente una hornacina grande y posteriormente achicada en su parte baja colocando dos hiladas de adobe hasta lograr un tamaño casi igual a las demás.

Materiales Constructivos.- La tierra, el canto rodado y el junco son los principales materiales utilizados diferenciadamente por las diversas formaciones sociales en los distintos períodos de la historia prehispánica de la región. Esta utilización de materiales constructivos ha permitido el desarrollo paulatino de formas simples a formas complejas haciendo de la arquitectura de barro la característica más resaltante de la ocupación local. La arquitectura de barro mas temprana registrada por D. Wallace corresponde a unos muros cuyos adobes son del tipo denominado "grano de maíz" por la forma ligeramente cónica que tienen. Se los encuentra asociados a los basurales ParacasCavernas, definiendo en algunos casos paredes de casas y de terrazas de montículos ceremoniales (Wallace 1971: 17).

En algunos casos se encuentran asociados a construcciones del Intermedio Tardío, sin embargo, en el período de los Estados Regionales desaparece totalmente en el valle y el "adobito" es reemplazado por la tapia como elemento característico y masivo de las construcciones. Aparentemente esta modalidad se mantuvo sin ninguna alteración hasta la conquista del valle por los Inkas que utilizaron el barro transformando en adobes de forma rectangular. La producción y uso del adobe se tornan masivos en las construcciones.
Si bien las descripciones hechas líneas arriba son las modalidades predominantes, tanto en el contexto Inka como en el local, encontramos que algunos sectores de ocupación local I, II, V, VI y el sector XI de ocupación Inka-Chincha evidencian construcciones hechas de adobe y tapia. Hemos logrado identificar tres variantes en el uso de estos elementos en las construcciones de los muros. Estos son: 1.- Tapia sobre adobe, 2.- Adobe sobre tapia y 3.- Tapia-adobe-tapia (horizontal).

La primera variante corresponde a los muros de cerramiento del sector XI, de ocupación bilateral. En el segundo caso, evidenciado en el sector I, encontramos que los adobes que rematan los muros de tapia son pequeños y de forma ligeramente rectangular. Al parecer se trata de un elemento adquirido o impuesto por los Inkas y construido por gente local un poco tardíamente a las construcciones de tapia.

La tercera variación corresponde al parecer, al uso del adobe exclusivamente para resanar algunas partes deterioradas de los muros.

\section{BiBLIOGRAFIA}

DOLLFUSS, Oliver

1981 "El Reto del Espacio Andino". IEP. Perú Problema $\mathrm{N}^{\circ}$ 20, Lima.

KROEBER, Alfred y William D. STRONG

1924 "The Uhle Collection form Chincha". Univ. of Calif. Public. In American Archaeology and Ethnology; Vol. 21, Berkeley.

LUMBRERAS, Luis

1974 "Los Reynos Post-Tiwanaku en el área Altiplánica". En Revista del Museo Nacional; $N^{\circ} 40$, Lima.

MENZEL, Dorothy

1959 "The Inka Occupation of the South Coast of Peru"; Southwestern Journal of Anthropology; Vol. 125 (2).

1966 "The Pottery of Chincha", Nawpa Pacha $\mathrm{N}^{\circ} 4$.

1976 Pottery Style and Society in Ancient Peru. Univ. of Calif. Press.

MENZEL, Dorothy y John ROWE

1966 "The Role of Chincha in Late Pre-Spanish Peru". Nawpa Pacha $\mathrm{N}^{\circ} 4$.

MORRIS, Craig

1974 "Asentamientos Estatales en el Tawantinsuyu: Una Estrategia de Urbanismo Obligado”. Revista del Museo Nacional. Lima. 
MORRIS, et al.

s/f "Ecological Complementarity and Long Distance Maritime Exchange in the Chincha Area, Peru", Ms.

ROSTWOROWSKI, María

1970 "Mercaderes del Valle de Chincha en la Epoca Prehispánica: Un documento y sus comentarios". Revista Española de Antropología Americana, $\mathrm{N}^{\circ}$ 5. Madrid.

1975 "Pescadores, artesanos y mercaderes costeños en el Perú Prehispánico”. Revista del Museo Nacional, $\mathrm{N}^{\circ}$ 41. Lima.

1977 Etnía y Sociedad: Costa Peruana Prehispánica. IEP, Lima.

1978 Señoríos Indígenas de Lima y Canta. IEP, Lima.

UHLE, Max

1924 "Explorations at Chincha". Univ. of Cal. Publ. En American Archaeology and Ethnology; Vol. 21; Berkeley.
WALLACE, Dwight

1959 "Informe del Reconocimiento del Valle de Chincha". En Revista del Museo Regional de Ica, $\mathrm{N}^{\circ} 10$.

1970 "Informe del Reconocimiento del Valle de Chincha”. Arqueología y Sociedad $\mathrm{N}^{\circ}$ 2.

1971 "Sitios Arqueológicos del Perú: Valle de Chincha y Pisco". Arqueologicas; $\mathrm{N}^{\circ} 13$, Museo Ncl. de Antropología y Arqueología, Lima.

1977 "Ceremonial Road in Chincha: Symbolic and Political Implications". Trabajo presentado en el Symposium de la SAA, New Orleans. 

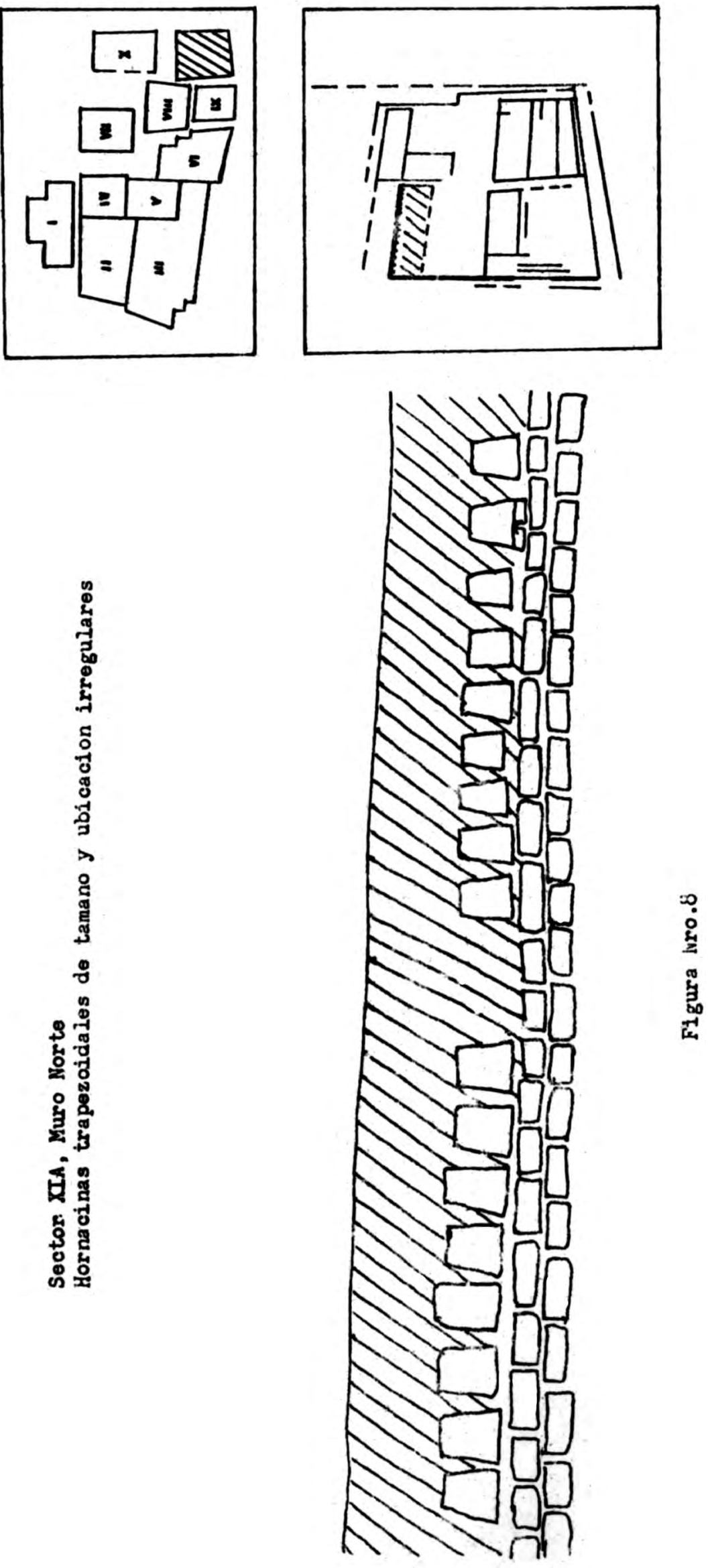
Variaciones en el uso del adobe en contextos locales y de ocupación bilateral

1'- VARLACION

THPIS/ $\triangle D O E=$

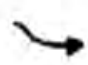

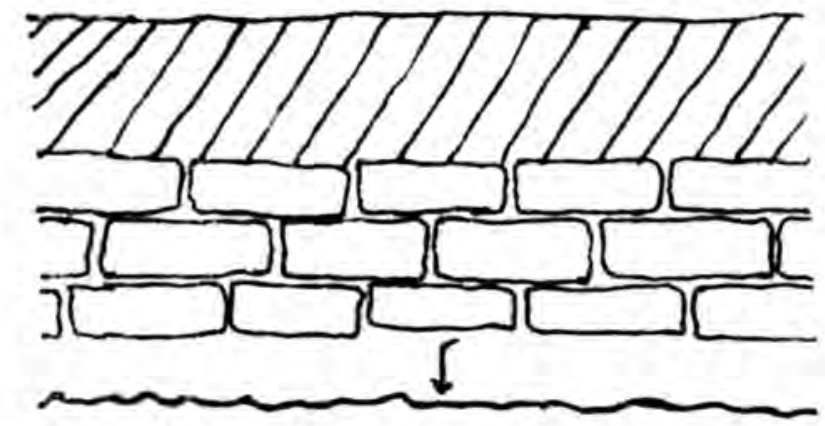

$2^{\text {da }} \cdot$ vasución usceer/TANA
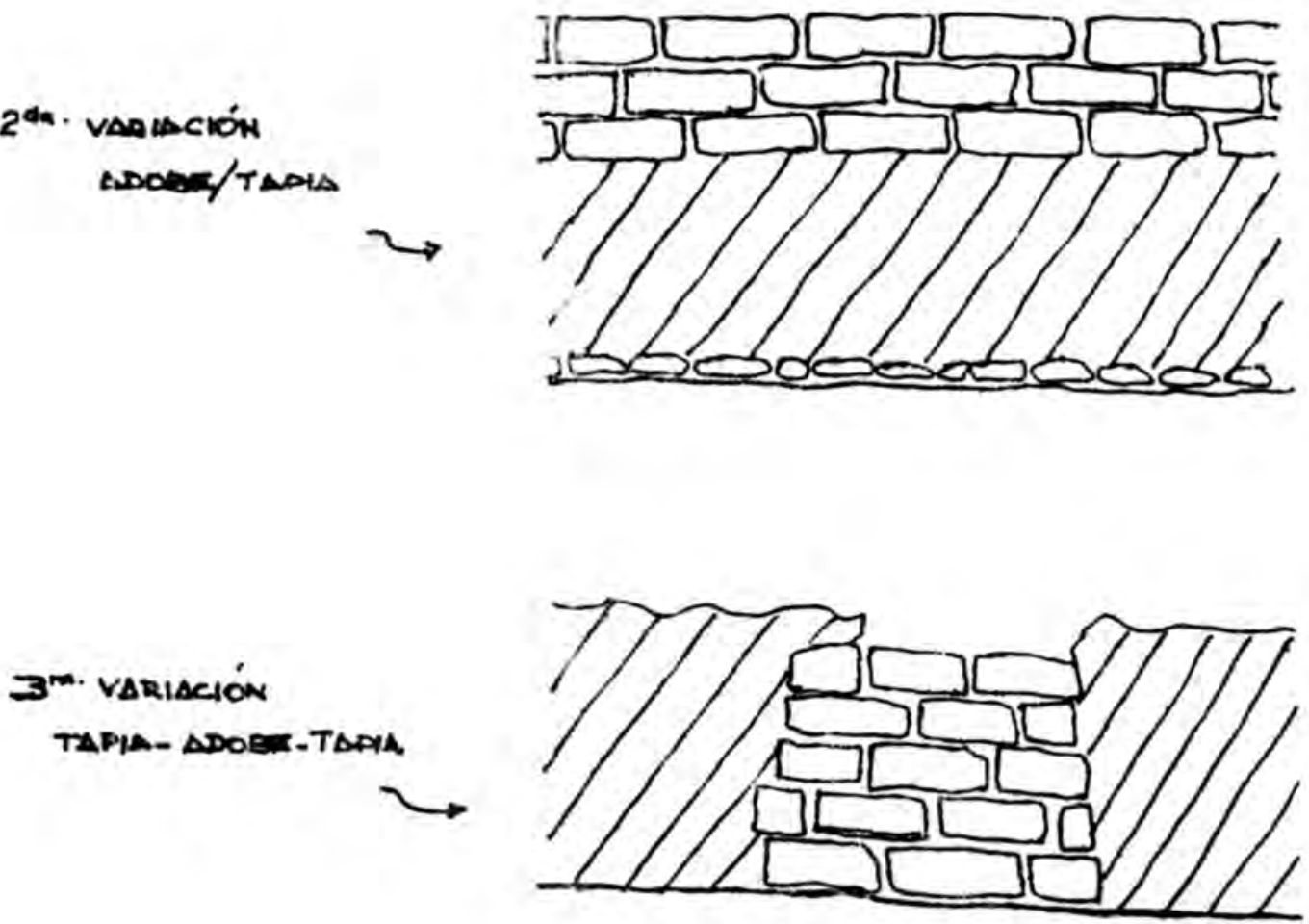

Bigura Nro.tl 


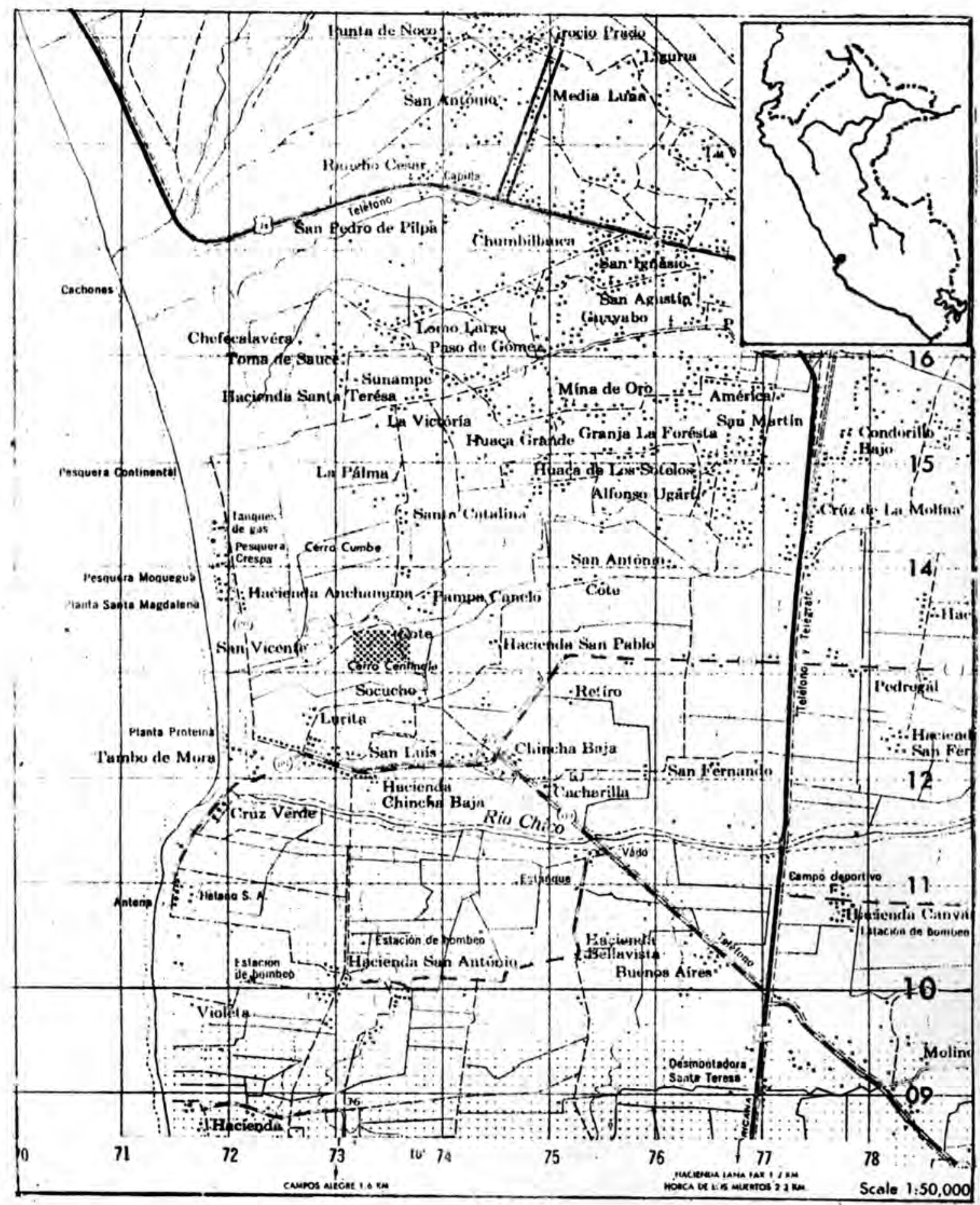

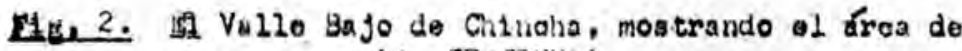
i.: CE. Ir.vient 


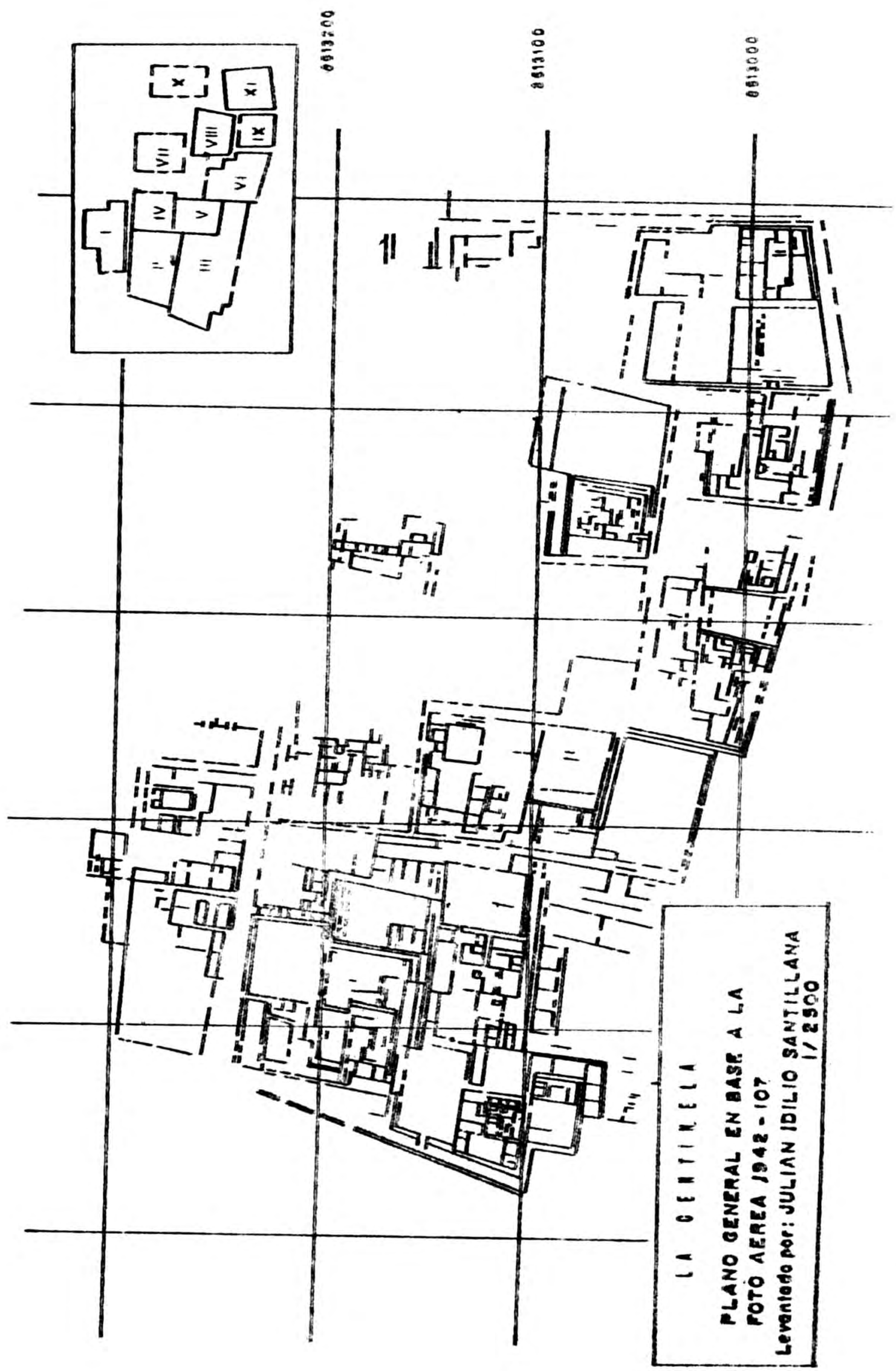



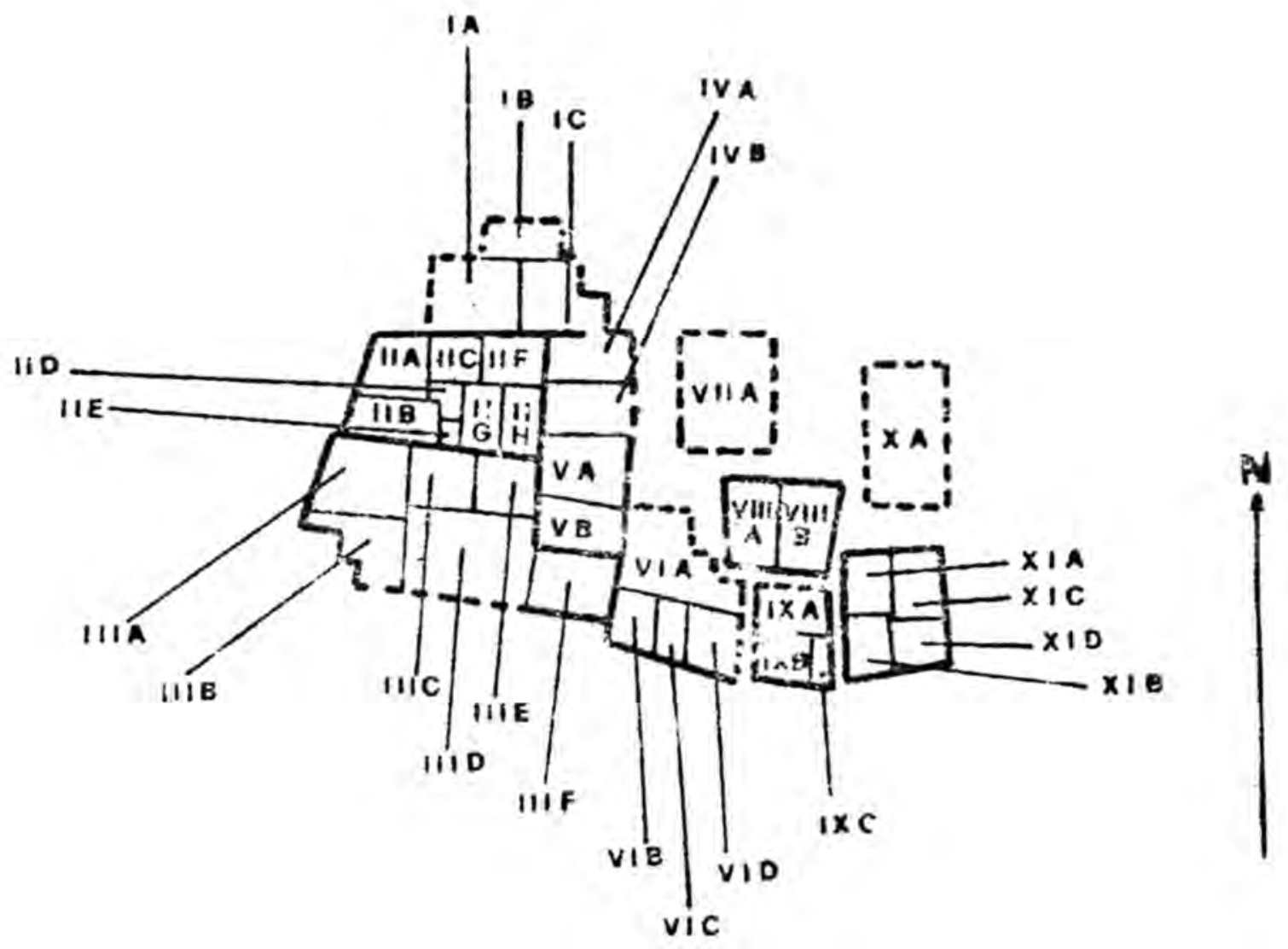
SUBSECTORIZACIÓN DEL COMPLEJO
LA CENTINELA (CH - C 10-1)
DIBUJADO SOBRE LA FOTOGRAFÍA AÉREA DE 1942 - HOJA 107-56 $1 / 10,000$

PLANO 3 
Formas generalizadas de los hornacinas Inka y Chinchanas

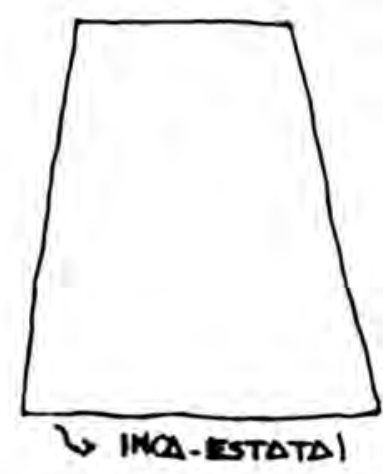

(a)

(b)
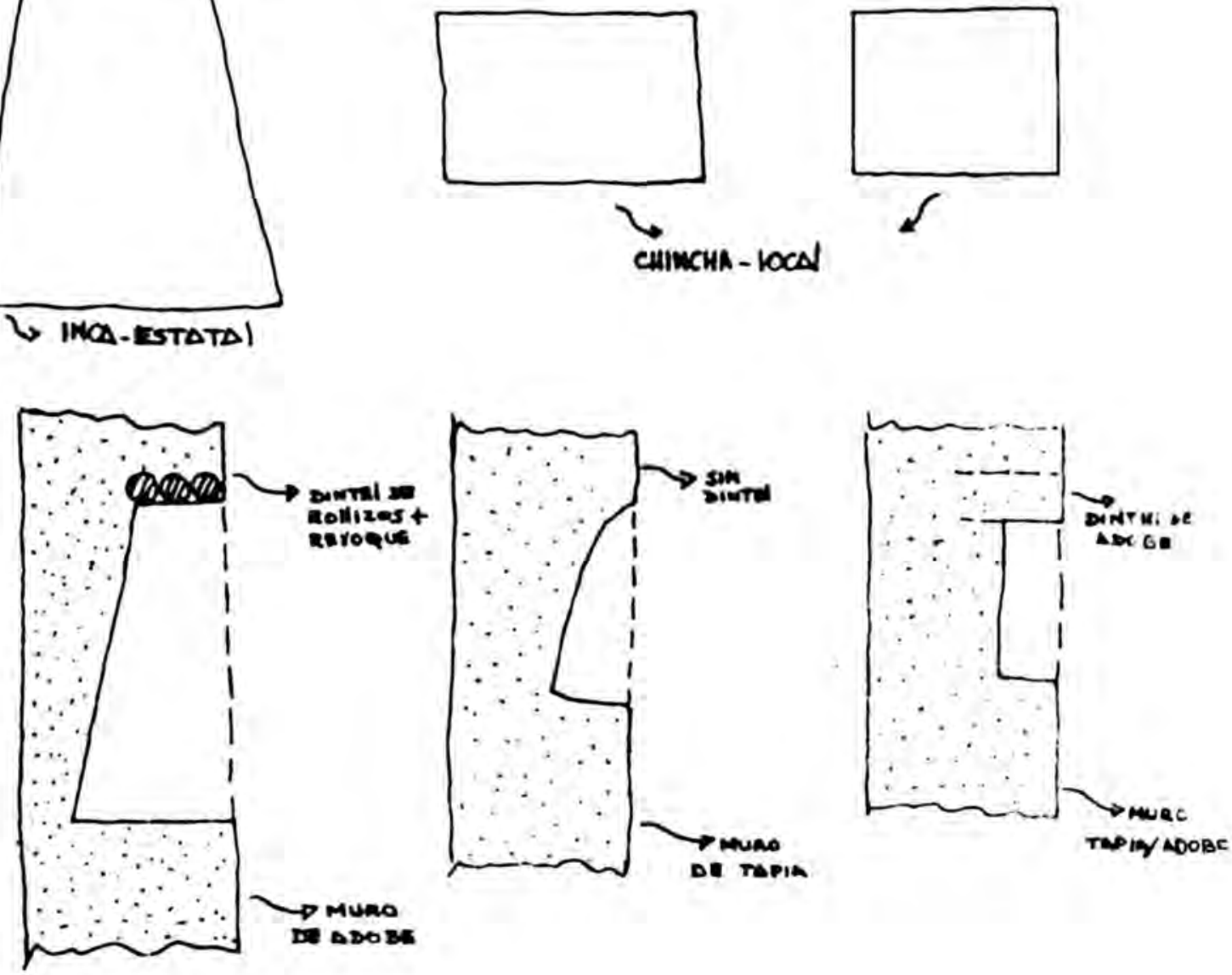

Figura 5 


\begin{tabular}{lcccc}
\hline Nro. & Muro & \multicolumn{3}{c}{ Dimensiones } \\
& & Largo & Ancho & Fondo \\
\hline 1 & Sur & 0.72 & 0.43 & 0.47 \\
2 & Sur & 0.75 & 0.31 & 0.22 \\
\hline
\end{tabular}

Características:

-Dimensiones no estandarizadas

-Forma trapezoidal con esquinas redondeadas

-Dintel de Adobe

- Acabado redondeado en su interior

-No presenta enlucido de barro

-Pintura blanca sobre el adobe

-Hecho después de terminado el muro

-Los materiales y algunos conceptos son Inka

\section{Graficos:}

1

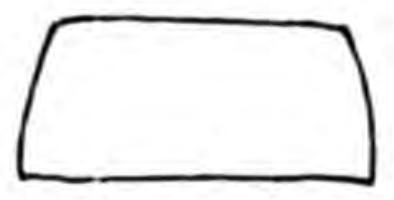

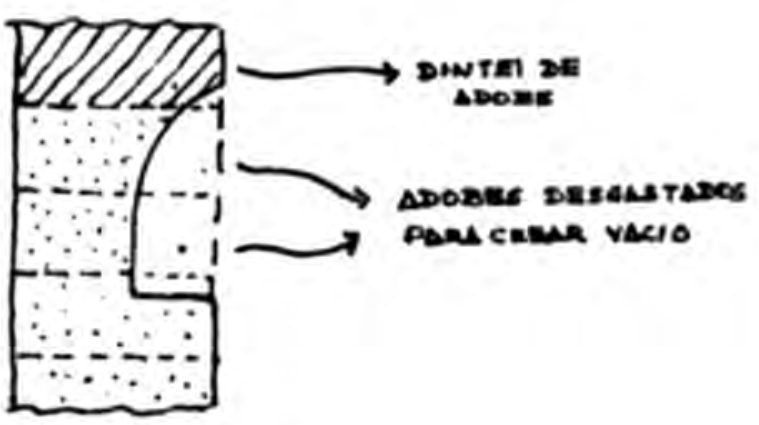

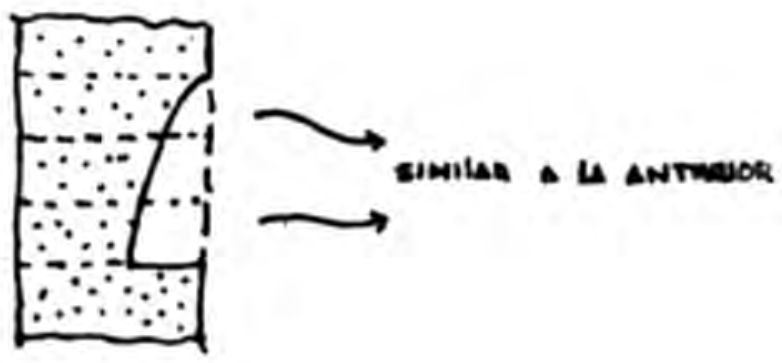

Figura Nro. 6 